\title{
The extremely high-energy electron experiment (XEP) onboard the Arase (ERG) satellite
}

Nana Higashio ${ }^{1,2^{*}}$, Takeshi Takashima ${ }^{3}$, Iku Shinohara ${ }^{3}$ and Haruhisa Matsumoto ${ }^{1}$

\begin{abstract}
The extremely high-energy electron experiment (XEP) onboard the Arase (ERG) satellite is designed to measure highenergy electrons in the Earth's radiation belts. The XEP was developed by taking advantage of our technical heritage of high-energy particle detectors that are onboard Earth observation satellites of the Japan Aerospace Exploration Agency (JAXA) as the radiation monitor. The main target of the XEP is to precisely measure variations of relativistic electrons in the outer radiation belt even during magnetic storms. The measurement is scientifically required to address physical mechanisms of electron acceleration and loss. The XEP consists of five solid-state silicon detectors (SSDs) and a single-crystal inorganic scintillator of cerium-doped gadolinium orthosilicate (GSO) to measure electrons in the energy range of $0.4-20 \mathrm{MeV}$ and has a $20^{\circ}$ single field of view (FOV). It is also equipped with a plastic scintillator that surrounds the GSO scintillator to prevent particles from entering the detectors from outside the FOV. The XEP has started its observation of relativistic electrons and has successfully observed dynamic variations of relativistic electron fluxes in the outer radiation belt during magnetic storms. This paper describes the instrumentation of the XEP and presents an example of initial observation results.
\end{abstract}

Keywords: ERG, Radiation belts, Extremely high-energy electron experiment

\section{Introduction}

Relativistic electrons are trapped in the Earth's radiation belts, and the integrated flux of these electrons is known to vary widely during geomagnetic storms (e.g., Miyoshi and Kataoka 2005). However, the physical mechanisms of the acceleration and loss of relativistic electrons remain unclear. Two types of electron acceleration processes have been proposed (e.g., Shprits et al. 2008a, b; Ebihara and Miyoshi 2011). One is adiabatic transportation caused by radial diffusion. The radial diffusion could possibly be attributed to the resonant interactions with the ULF (Pc4-5) waves and energetic electrons coming from the outer magnetosphere. The electron energy increases as electrons move toward a stronger magnetic field region (earthward) due to the conservation of the

\footnotetext{
*Correspondence: higashio.nana@jaxa.jp

${ }^{1}$ Research and Development Directorate, Japan Aerospace Exploration Agency, Tsukuba 305-8505, Japan

Full list of author information is available at the end of the article
}

first adiabatic invariant. The other type falls under the category of non-adiabatic acceleration. The wave-particle interaction process is thought to be essential for electron acceleration. The cyclotron resonance with whistler mode chorus waves is considered as the most important candidate process to explain electron acceleration in the outer radiation belt (e.g., Summers et al. 1998; Miyoshi et al. 2003).

The Exploration of energization and Radiation in Geospace (ERG) is a science mission to study electron acceleration and loss mechanisms in the outer radiation belt. The "Arase" (ERG) satellite was developed by the Japan Aerospace Exploration Agency (JAXA) in collaboration with universities and institutes in Japan and Taiwan. The essential key observation of this program entails using the Arase satellite to conduct detailed in situ measurements of particles and electromagnetic fields in the radiation belts. Arase was launched on December 20, 2016, from the Uchinoura Space Center (USC) located in the southern part 
of Kyushu and initiated its scientific observation on March 24, 2017. Arase is designed as a spin-stabilized satellite with a rotation rate of about $7.5 \mathrm{rpm}$. Given its perigee altitude of about $400 \mathrm{~km}$ and its apogee altitude of about 32,000 km, Arase can cover the entire radiation belts in its orbit. Its inclination is about $31^{\circ}$. The orbital period is about $570 \mathrm{~min}$.

Four electron sensors are onboard Arase to cover a wide energy range of $19 \mathrm{eV}-20 \mathrm{MeV}$, and the extremely high-energy electron experiment (XEP) is responsible for measuring the highest energy part $(0.4-20 \mathrm{MeV})$. The XEP is required to accurately measure relativistic electron fluctuations in the outer belt, and it provides key science data in order to better understand the acceleration and loss processes of relativistic electrons in the radiation belts during magnetic storms. The $\mathrm{XEP}$ is also expected to contribute the cross-calibration with the high-energy electron experiment (HEP) which measures high-energy electrons of $0.07-2 \mathrm{MeV}$ (Mitani et al. 2018). The cross-calibration between the XEP and the HEP enables us to improve the accuracy of both measurements.

The XEP was developed by taking advantage of our technical heritage of high-energy electron sensors, such as electron sensors onboard the "Ibuki" (GOSAT) satellite, which has been in operation for more than 9 years (e.g., Yasutomo et al. 2009), and it can measure high-energy electrons in a wider energy range with higher energy resolution and higher time resolution as compared with the onboard electron sensors of Ibuki.

\section{Description of instruments}

\section{Instrument design and detection unit}

Figure 1 shows (a) a photograph and (b) a cross-sectional view of the XEP flight model. As shown in Fig. 1b, the detection unit is tilted $10^{\circ}$ from the vertical to prevent other onboard instruments from entering the field of view (FOV).

Figure 2 shows a cross-sectional view of the detection unit. The collimator consists of four aluminum cylinders $(6 \mathrm{~mm}$ in inside diameter) and two tantalum cylinders $(8 \mathrm{~mm}$ in inside diameter) and has a total length of $18.5 \mathrm{~mm}$. Given the $20^{\circ} \mathrm{FOV}$, the geometrical factor of the XEP evaluated by the numerical model is $0.0088 \mathrm{~cm}^{2}$ sr.

The XEP measures high-energy electrons in the energy range of $0.4-20 \mathrm{MeV}$, and the detection unit consists of five silicon solid-state detectors (MSD007-50: SSD-1, MSD018-1500: SSD-2-SSD-5 in Fig. 2) used to measure the energy range of $0.4-5.4 \mathrm{MeV}$ and a single-crystal inorganic scintillator of cerium-doped gadolinium orthosilicate (GSO: indicated as GSO scintillator in Fig. 2) that is located behind the SSDs in order to measure the energy range of 6-20 MeV. Light emissions from the GSO scintillator are detected by using a photomultiplier (HAMAMATSU, R3991A-04: PMT in Fig. 2). An aluminum shield ( $130 \mu \mathrm{m}$ thick) is placed in front of the first SSD to prevent the intrusion of light and electrons with the energy of less than $0.4 \mathrm{MeV}$.

The first SSD (SSD-1 in Fig. 2) is $50 \mu \mathrm{m}$ thick, while the other four SSDs are $1500 \mu \mathrm{m}$ thick. The appearance of the GSO scintillator is in the shape of a cylinder $(20 \mathrm{~mm}$ in diameter, $20 \mathrm{~mm}$ in length). As shown in Fig. 2, to prevent
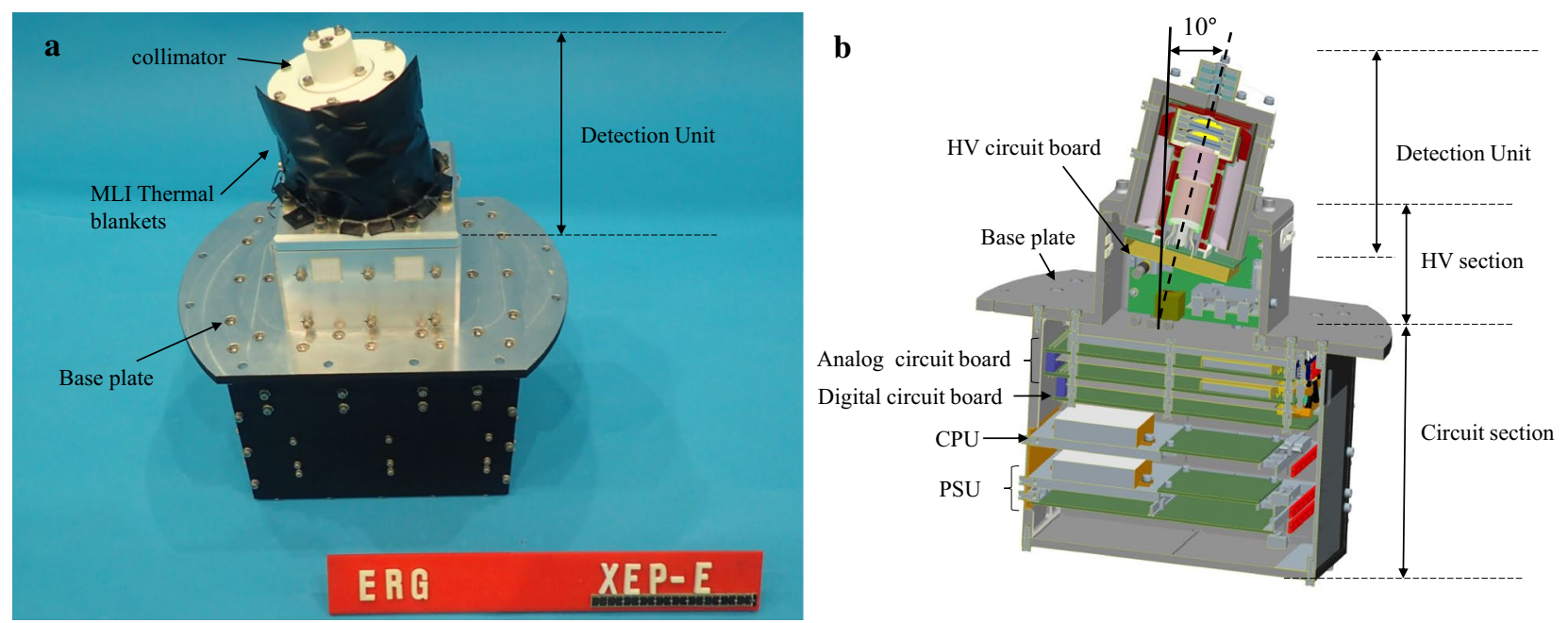

Fig. 1 a A photograph and $\mathbf{b}$ a cross-sectional view of the XEP flight model. The part exposed outside the spacecraft body is covered by multilayer insulation (MLI) blankets, and the detection unit is partially painted white for cooling 


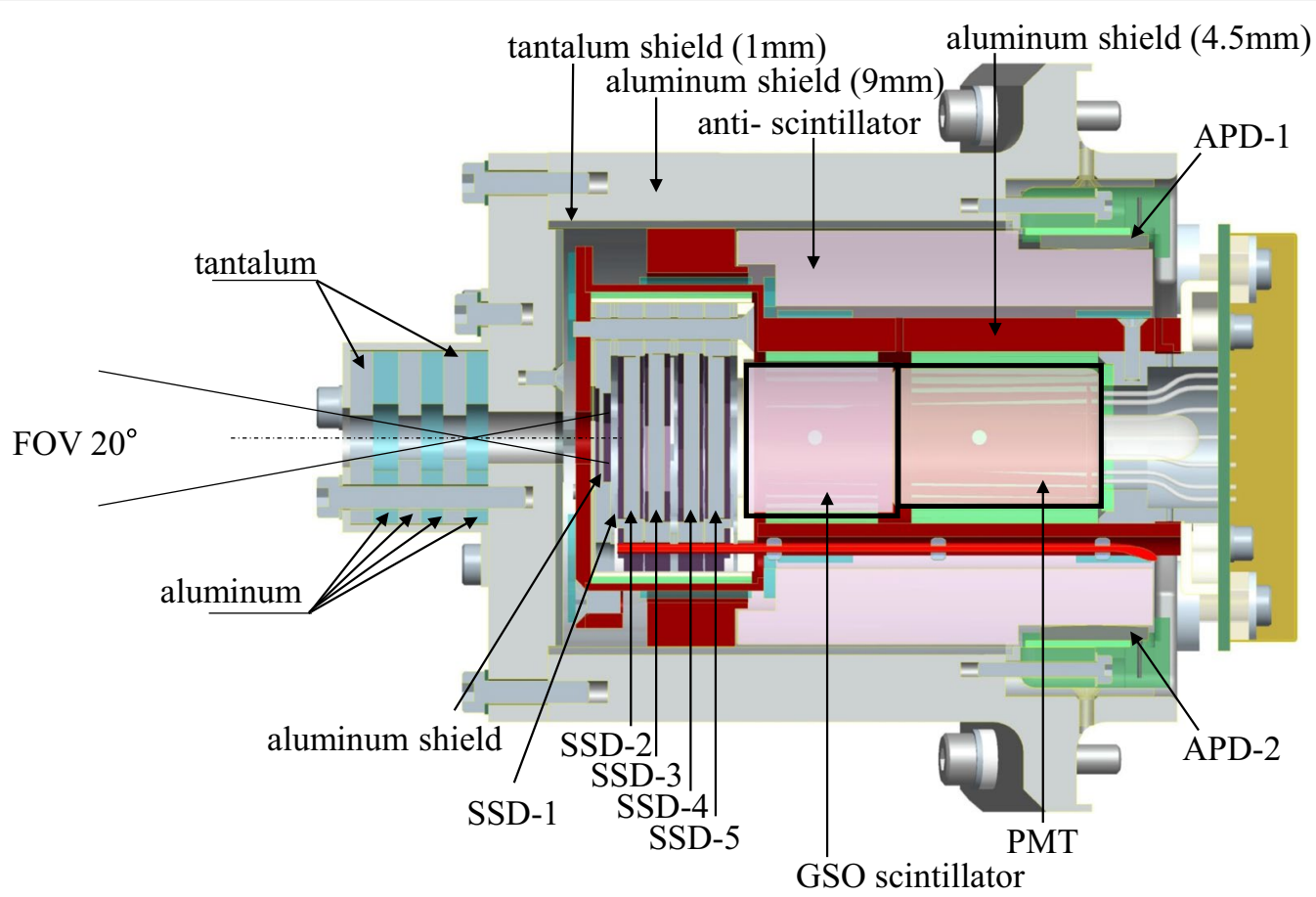

Fig. 2 A cross-sectional view of the XEP's detection unit (flight model)

contamination by particles from entering the detectors from outside the FOV, an aluminum shield ( $9 \mathrm{~mm}$ thick) and a tantalum shield ( $1 \mathrm{~mm}$ thick) cover the SSDs and the scintillator. An anti-scintillator (a plastic scintillator $10 \mathrm{~mm}$ thick) also covers the GSO scintillator to detect particles penetrating through the thick shield. Two avalanche photodiodes (APDs; S8664-1010) are used for detecting the signals from the anti-scintillator, instead of the photomultiplier to reduce the overall weight of the XEP. About $85 \%$ of electrons under $9 \mathrm{MeV}$ stop inside the aluminum and tantalum shields, and the counting rate of electrons at the anti-scintillator increases as their energy becomes higher. When the anti-scintillator detects signals, corresponding incident signals are not inputted to the analog-to-digital conversion process.

Since the performance of the APDs is severely influenced by the temperature condition, the APD temperature must be kept between -30 and $+45{ }^{\circ} \mathrm{C}$, and the thermal control design is required for the XEP. The detection unit is partially painted white for cooling and thermal insulation from the high-voltage unit area. As the temperature of the detection part is lowered by heat radiation, the sensor head is painted white and covered with multilayer insulation (MLI) blankets, and the XEP temperature is controlled between -20 and $45{ }^{\circ} \mathrm{C}$ with a heater mounted on the base plate, so as to maintain a constant temperature of APDs as much as possible. As the gain of the APDs varies depending on the temperature, the high-voltage power supplied to the APDs is controlled based on the APD temperature to keep the APDs gain constant. The platinum temperature sensor placed near the APDs measures the reference temperature of APDs, and the measured temperature is used for the gain control.

Two analog circuit boards, one digital circuit board, one central processing unit (CPU) and one power supply unit (PSU) are located under the base plate inside the satellite as shown in Fig. 1b. The circuit section will later be described in detail. Table 1 summarizes the specifications of the XEP, including energy range and resolution, types of data, size, weight and power consumption.

A detailed numerical simulation of the XEP detector responses to electrons and protons was performed using GEANT4 (GEometry ANd Tracking 4) to establish the XEP's detector design. All the major parts of the XEP (the collimator, the shielding (Al: $9 \mathrm{~mm}$ and Ta: $1 \mathrm{~mm}$ ), the aluminum film in front of the first SSD, the SSDs, the GSO scintillator, the PMT, the anti-scintillator and the aluminum shielding ( $4.5 \mathrm{~mm}$ thick) between the GSO scintillator and the anti-scintillator) were modeled in this simulation. In order to make it easy to compare the results of the numerical simulation with irradiation tests, all the particles were injected perpendicular to the surface of the SSDs. 
Table 1 The XEP specifications

\begin{tabular}{|c|c|c|}
\hline Parameter & Value & Notes \\
\hline Sensor & Extremely high-energy electron experiment & \\
\hline Type of measurement & Electron & \\
\hline \multirow[t]{3}{*}{ Energy range } & $0.4-20 \mathrm{MeV}$ & \\
\hline & $\triangle E / E<8 \%$ (SSD region) & FWHM \\
\hline & $\Delta E / E<60 \%$ (main scintillator region) & FWHM \\
\hline Geometry factor & $0.0088 \mathrm{~cm}^{2} \mathrm{sr}$ & $\begin{array}{l}\text { Calibrated in } \\
\text { the numerical } \\
\text { model }\end{array}$ \\
\hline FOV & $20^{\circ}$ & \\
\hline Spin Phase & 16 phases/spin & \\
\hline \multirow[t]{3}{*}{ Type of data } & Table mode (16ch)/list mode & \\
\hline & List mode (for S-WPIA) & \\
\hline & Table mode (for Space Weather) & \\
\hline Dimension & $317 \mathrm{~mm} \times 250 \mathrm{~mm} \times 174 \mathrm{~mm}$ & \\
\hline Weight & $5281 \mathrm{~g}$ & \\
\hline Power consumption & $16.7 \mathrm{~W}$ & \\
\hline
\end{tabular}

We define the peak energy $(E)$ as the energy at the maximum probability of the deposited energy spectrum obtained from the constant energy electron incidents. The energy resolution is defined as $\Delta E / E$. Here, $\Delta E$ is the full width at half maximum (FWHM) of the deposited energy spectrum. Figure 3 shows examples of the simulation results, (a) the case of $1 \mathrm{MeV}$ electron incident and (b) $14 \mathrm{MeV}$ electron incident.

Figure 4 shows the GEANT4 simulation results of energy resolution. The results indicate that electrons up to $\sim 5 \mathrm{MeV}$ are stopped in the SSD region, and the XEP can observe these electrons $(0.4-5 \mathrm{MeV})$ with the energy resolution of less than $8 \%$ at $0.4 \mathrm{MeV}$. The results also show that electrons of $6-20 \mathrm{MeV}$ are stopped not only in the SSD region but also in the GSO scintillator region, and the XEP can observe these electrons $(6-20 \mathrm{MeV})$ with the energy resolution less than $60 \%$ at $20 \mathrm{MeV}$. There is an energy gap in the measurable energy range of the XEP, and the gap corresponds to the energy range between the measurable ranges of the SSDs and the GSO scintillator. Electrons less than $6 \mathrm{MeV}$ cannot enter the GSO scintillator easily due to the strong backscattering that occurs on its entire surface. The energy resolution in the GSO scintillator is worse than that of the SSDs because some portion of the incident electron energy is converted to the gamma-ray emission and the probability
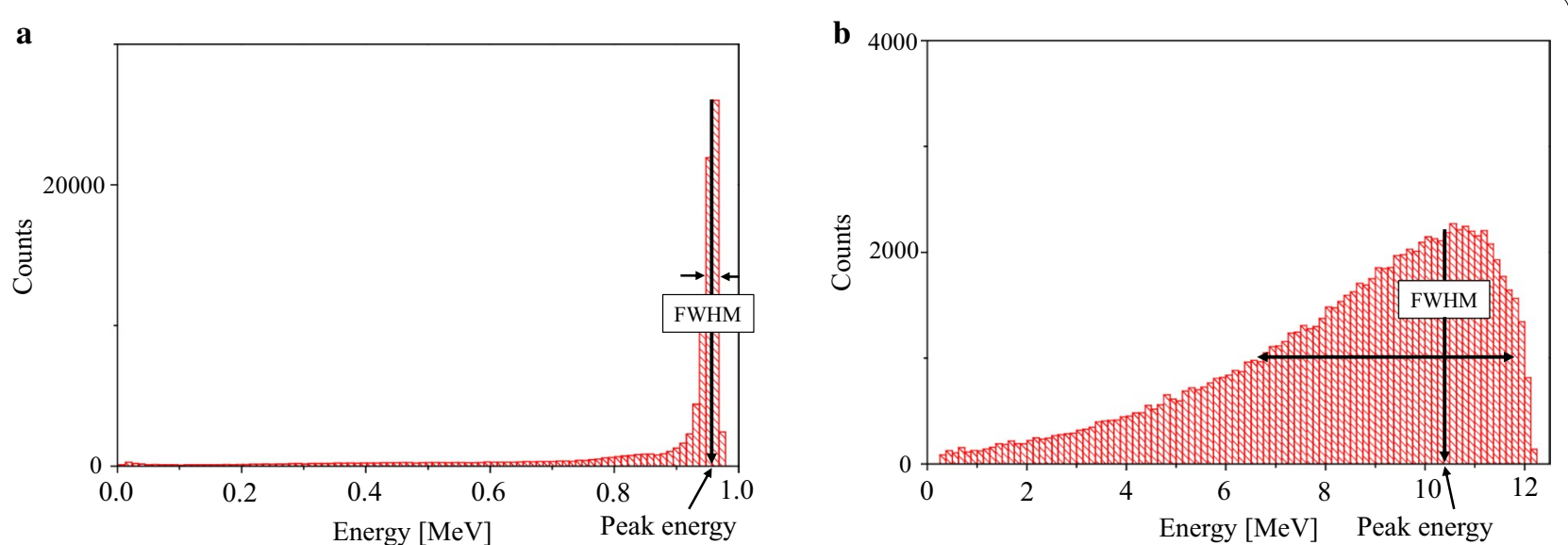

Fig. 3 Examples of the deposited energy spectra obtained by the GEANT4 simulation: $\mathbf{a}$ a deposited energy spectrum of the SSD region in the case of $1 \mathrm{MeV}$ electron incident and $\mathbf{b}$ a deposited energy spectrum of the GSO scintillator region in the case of $14 \mathrm{MeV}$ electron incident 

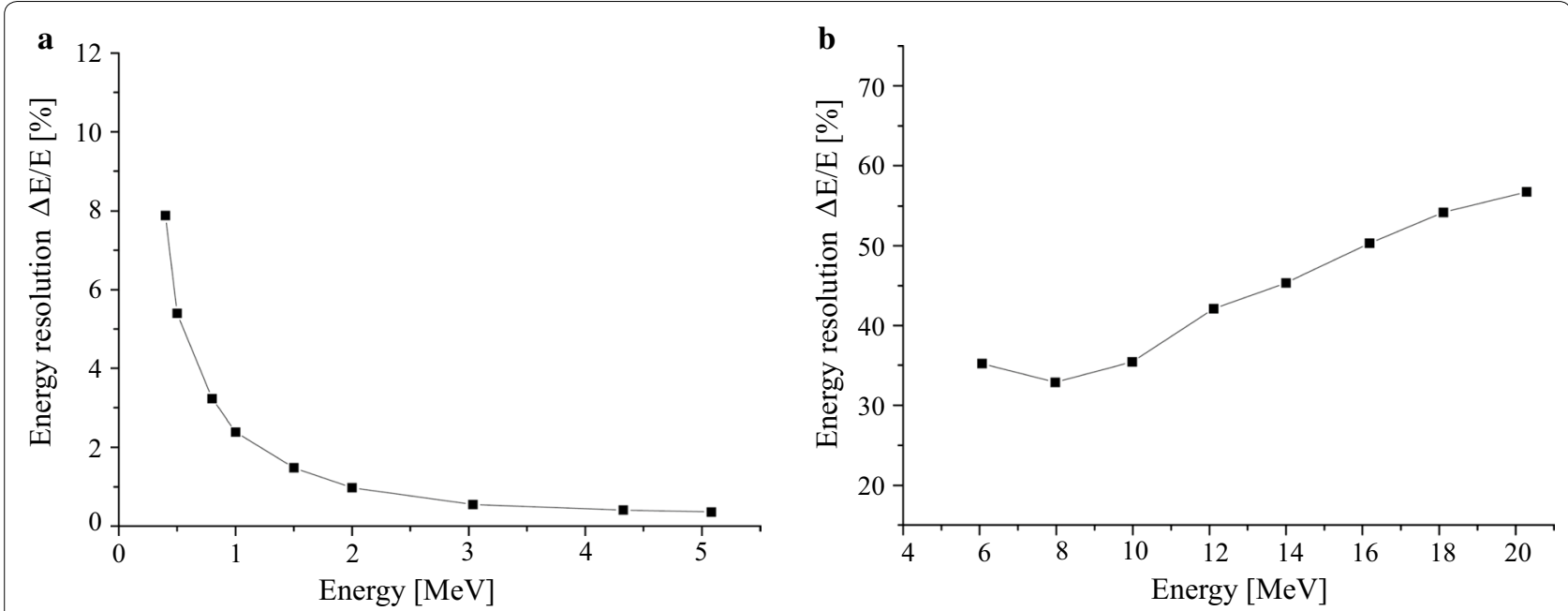

Fig. 4 The energy resolution estimated from the GEANT4 simulation results. a The energy resolution of the SSDs and $\mathbf{b}$ the energy resolution of the GSO scintillator

of electrons $(>6 \mathrm{MeV})$ reaching the GSO scintillator is lower. The probability of electrons over $10 \mathrm{MeV}$ reaching the GSO scintillator is $90 \%$, while the probability of $8 \mathrm{MeV}$ electrons is $77 \%$ and that of $6 \mathrm{MeV}$ electrons is $56 \%$.

\section{Analog electronics}

Figure 5 illustrates a block diagram of two analog circuit boards. Output signals from each detector are collected by each subsequent preamplifier (PREAMP), and the output of each preamplifier drives corresponding shaping

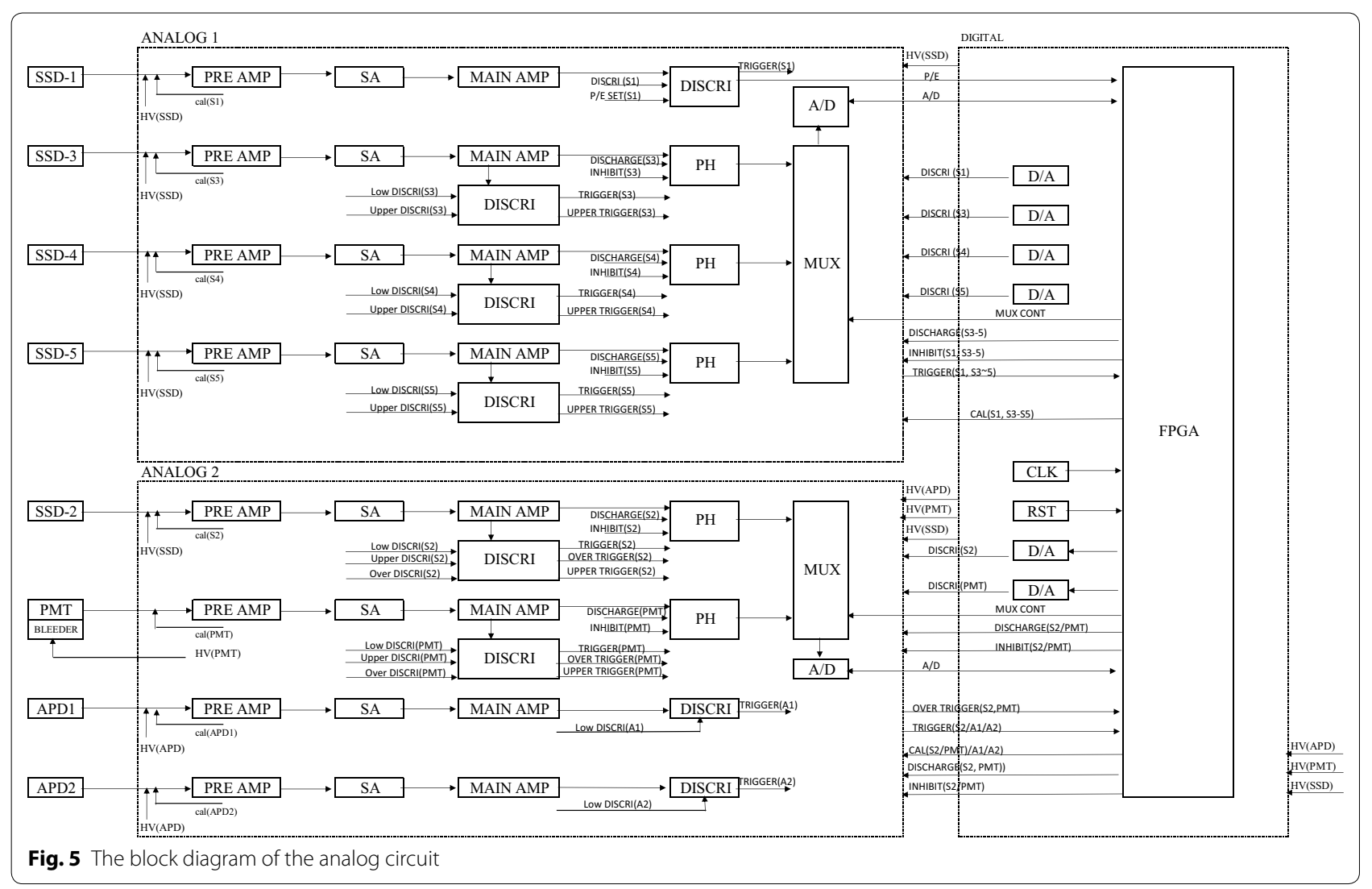


amplifier (SA). The peaking time of the SA is $1 \mu \mathrm{s}$. The output of each SA drives main amplifiers (MAIN AMP), and each MAIN AMP output is peak-held (PH). These outputs from the PHs are merged by a multiplexer (MUX), and then, the analog signals from the MUX are converted to digital signals by an analog-to-digital converter (ADC). Each signal after the ADC is sent to a fieldprogrammable gate array (FPGA).

Output signals from the MAIN AMPs of the first SSD (SSD-1) and two APDs (APD-1 and APD-2) are not peakheld $(\mathrm{PH})$. They are only used to discriminate particles during the onboard data processing. We use the output signals of four SSDs (SSD-2-SSD-5) and the main scintillator (+PMT) to determine the energy values of incident electrons. The MAIN AMP outputs of SSD-1 and SSD-2 are used to judge whether to convert the $\mathrm{PH}$ signals of SSD-2 to SSD-5 and the main scintillator (+ PMT) by the ADC.

The data processing time is $13.175 \mu$ s. (The $\mathrm{PH}$ window time $=3 \mu \mathrm{s}$, the $\mathrm{A} / \mathrm{D}$ conversion time $=3.675 \mu \mathrm{s}$, the $\mathrm{PH}$ discharge time $=4.5 \mu \mathrm{s}$ and the dead time for self-transmission $=2 \mu \mathrm{s}$.) When the XEP does not perform the $\mathrm{A} / \mathrm{D}$ conversion process in an event, its processing time is $9.5 \mu$ s (with $\mathrm{PH}$ window time of $3 \mu \mathrm{s}$, PH discharge time of $4.5 \mu \mathrm{s}$ and dead time for self-transmission of $2 \mu \mathrm{s}$ ). The XEP counts the dead time in units of 25 ns. This dead time information is necessary for converting raw count data to the physical quantities.

\section{Discrimination}

Three types of discriminations, lower discrimination (LD), upper discrimination (UD) and over discrimination (OD), are prepared to extract electron incident events. Each discrimination value can be set by commands. As shown in Fig. 5, the LD is set for all signal channels, and the HD is set for all signal channels except for $\mathrm{S} 1$ and the APDs. The OD is set for SSD-2 and the GSO scintillator (+ PMT).

In the cases of SSD-2 to SSD-5, signals detected between the LD and the UD are processed as incident events. The XEP is designed to be able to discriminate between protons and electrons during the onboard data processing. We implemented this function as the proton/ electron mode (the $p / e$ mode). For SSD-1, only the LD is set, and it discriminates between protons and electrons by utilizing the difference in the energies deposited to SSD-1 due to protons and electrons. We judge that signals beyond the LD of SSD-1 are due to protons to be measured. A threshold value of the LD for SSD-1 is set as the deposited energy of the $0.4 \mathrm{MeV}$ electron to discriminate between the deposited energies of electrons and of protons. A threshold value of the LD for SSD-2 is set as the deposited energy of the $0.2 \mathrm{MeV}$ electron to discriminate electrons. The discrimination results of SSD-1 and SSD-2 are used to determine the triggering of the A/D conversion to eliminate protons.

The dead time may be longer due to the mixing of particles that drop excessive electric charges in such detection parts as protons and heavy ions, resulting in fewer events that should be acquired. In order to solve this problem, OD is set for SSD-2 and the GSO scintillator (+PMT). When a signal is detected by the OD, an appropriate inhibit time (of $153 \mu \mathrm{s}$ ) is set for analog signals until the discharge due to excessive input is completed. For APD-1 and APD-2, only the LD is set as the anti-scintillator detectors, and the value of the LD is set slightly higher than the noise level. All signals exceeding the LD are determined to be particles not subject to observation, and the A/D conversion for such events is not triggered. The electron energies detected in the scintillator region are determined only by signals in the scintillator region after the conditions are satisfied in the FPGA (i.e., the total energy value of the SSDs is between $1.5 \mathrm{MeV}$ and $2.5 \mathrm{MeV}$, the LD information of SSD-5 and the GSO scintillator).

\section{Digital electronics}

The XEP uses a field-programmable gate array (FPGA; RTAX2000) and a central processing unit (CPU) for the digital processing of the A/D converted signals. The FPGA plays many roles, such as the management of the high-voltage control and the discrimination control, mission data processing, and the generation of trigger information and telemetry data including mission data and housekeeping data.

$+12 \mathrm{~V},-12 \mathrm{~V}$ and $+3.3 \mathrm{~V}$ are supplied from the power supply unit (PSU), and it consumes about 16.7 W. The XEP uses three high-voltage units, $-1020 \mathrm{~V}$ for the photomultiplier (PMT), $-320 \mathrm{~V}$ for the five solid-state silicon detectors (SSDs) and $-360 \mathrm{~V}$ for the two avalanche photodiodes (APDs). Voltages are slowly changed over the time of $60 \mathrm{~s}$ to step up and to step down the high-voltage power supply under the FPGA control. With reference to a temperature near a point of the APDs, the high voltage is automatically adjusted according to a correspondence table of temperatures and high voltages memorized in the FPGA register. The CPU board is located behind the digital circuit board. The CPU receives data from the FPGA and sends it to the system data recorder that records all telemetry data generated in the satellite.

The XEP has two data modes, the table mode and the list mode, and it generates three types of mission data, (1) normal science data of the table and list modes, (2) science data of the list mode for the software-type waveparticle interaction analyzer (S-WPIA data) (Katoh et al. 2018; Hikishima et al. 2018) and (3) science data of the 
table mode for space weather applications (SW data). It also generates housekeeping data and responses to operation commands.

The table mode is used to count numbers of incident events in each preset energy channel according to signals from the $\mathrm{ADC}$ and to make histograms of incident electrons depending on their energies. As listed in Fig. 6b, the table mode prepares 16 energy channels for the normal science data. The ch0-11 are used for events detected in the SSD region, and ch12-15 are for events detected in the GSO scintillator region. The energy resolution with the energy bins listed in Fig. $6 \mathrm{~b}$ is $\sim 5.4 \%$ in the SSD region, and the energy resolution in the GSO scintillator region is $10-56 \%$. However, the energy bins are variable by commands, and users should carefully check the Wiki page for the XEP information (https://ergsc.isee.nagoy a-u.ac.jp/mw/index.php/ErgSat/Xep).

The table mode data are divided into 16 phase angle bins of $22.5^{\circ}$ angular resolution according to the spacecraft spin. The spacecraft spin period is determined using the sun pulse signal from the spacecraft system, and the time interval of each phase is calculated from the previous spin period (Fig. 6a).

The science data of the list mode include the $\mathrm{PH}$ values of SSD-2-SSD-5 and the GSO scintillator of each event to get detailed event information, and the data rate is 1000 events/sec. The list mode data for S-WPIA include the
$\mathrm{PH}$ values of the sum of SSD-2-SSD-5 and the GSO scintillator of each event, and the data rate is 1024 events/sec.

The SW data are aimed at acquiring observation data in almost real time though they are available only during the real-time operation. The total amount of the SW data is limited to realize the real-time transfer so that the SW data are generated by reducing the normal science data of the table mode into six channels. The SW data are distributed to users in almost real time from the Space Environment and Effects System (SEES) of JAXA (http://sees. tksc.jaxa.jp/fw/dfw/SEES/index.html).

The telemetry size of the normal science data of the table mode is 1520 bytes per spin. The telemetry data of the normal science data include numbers of counts in each channel and spin phase, numbers of each discrimination (the LD, the UD and the OD) and a number of the dead time. The telemetry size of the SW data is 264 bytes per spin. The telemetry data of the SW data include numbers of counts in each channel.

\section{Calibration by irradiation test}

In order to determine the relationship between ADC channels and the absolute energy values, we carried out irradiation tests using the energies of which are well defined by facilities. The irradiation tests for the calibration of $0.4-2 \mathrm{MeV}$ electrons were performed at the Tsukuba Space Center (TKSC) (http://sees.tksc.jaxa.jp/

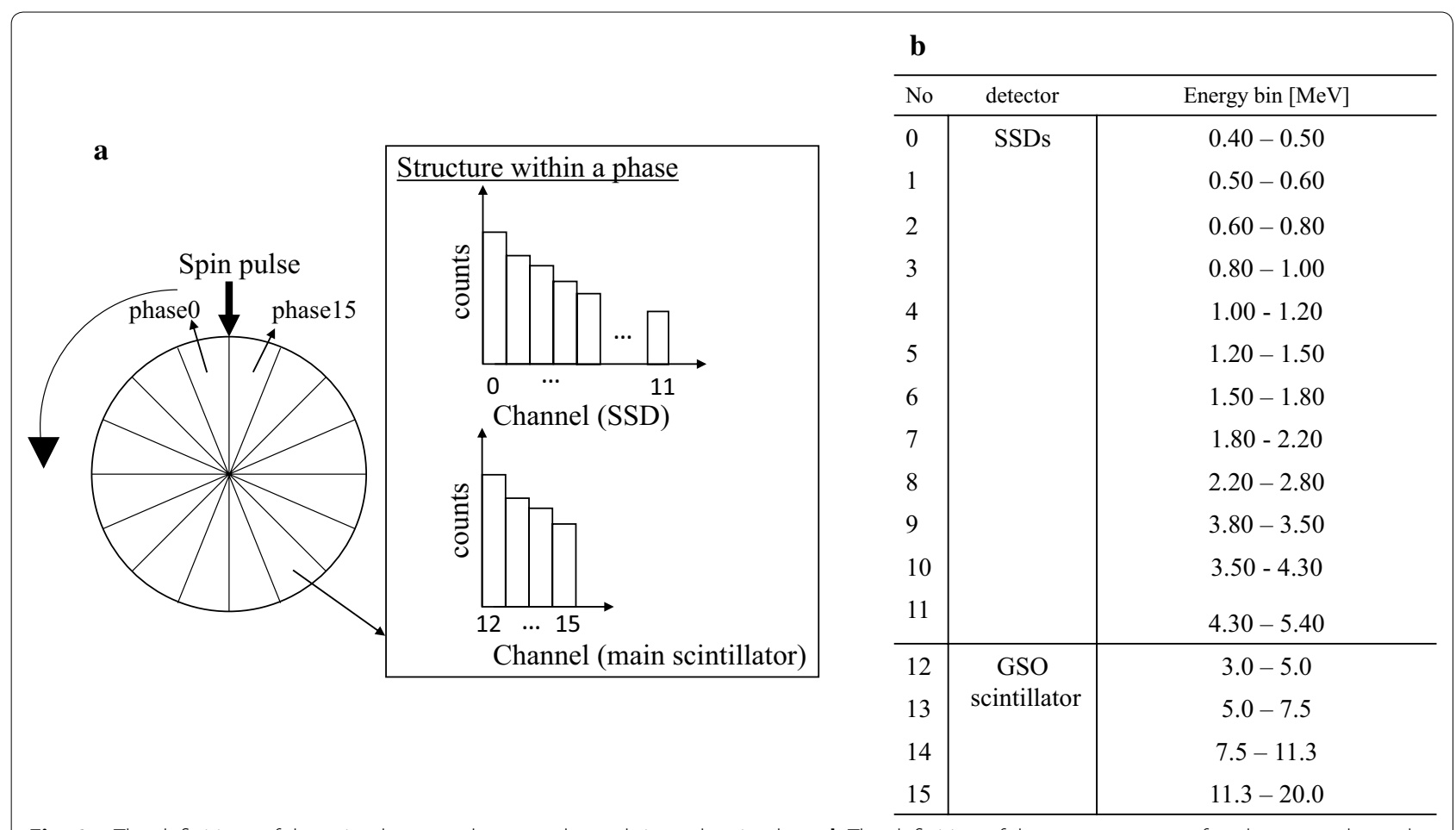

Fig. 6 a The definitions of the spin phases and energy channels in each spin phase. $\mathbf{b}$ The definition of the energy ranges of each energy channel 
fw_e/dfw/SEES/English/Labo/labo_e.shtml), and the irradiation tests for the calibration of $6-18 \mathrm{MeV}$ electrons were carried out by using the electron linear accelerator at the Kyoto University Research Reactor Institute (KURRI) (http://www.rri.kyoto-u.ac.jp/en/facilities/ela). Irradiation tests at TKSC were conducted in a vacuum chamber, but irradiation tests conducted at KURRI were done in the air because no vacuum chamber was available for the XEP. The energy deposited in the air was estimated at several tens of $\mathrm{keV}$, and it is ignorable comparing with the energy deposited to the GSO scintillator.

Figure 7 shows the results of the irradiation tests and the GEANT4 simulation. Note that the results of the irradiation tests are already converted from the ADC channels to the absolute energy by multiplying conversion factors. The conversion factors are determined by the linear fittings of the TKSC irradiation test results to the GEANT4 simulation results. As can be seen in Fig. 7a, both of the TKSC irradiation tests and the simulation results show a clear linear relation in the SSD energy range, and it indicates that the conversion factors to interpret the ADC channels as the absolute energies are well determined.

In the energy range of the GSO scintillator, the energy deposited to the gamma-ray emission increases as the incident energy becomes higher. Therefore, it was inferred from the GEANT4 result that the relation between incident energies and corresponding deposited energies is not linear. Figure $7 \mathrm{~b}$ presents the converted results of the KURRI irradiation tests for the energy range of the GSO scintillator. It suggests that the
GEANT4 simulation is also applicable to the absolute energy conversion of the ADC channels in the energy range of the GSO scintillator measurement since curves of the irradiation tests and the simulation results agree well. Although subtracting a small energy offset value is necessary for the conversion of the KURRI irradiation test results, the energy offset may come from environmental errors of the facility.

To simplify the onboard calibration process, the result of the GEANT4 simulation is fitted by a polynomial function, and this polynomial approximation is used. Since the difference between the polynomial approximation and the result of irradiation test is less than $10 \%$, we concluded that the polynomial approximation is applicable in the energy range of the GSO scintillator.

Figure 8 shows the energy resolution of each energy evaluated by the irradiation tests. Figure 8 a presents the resolution of the SSD energy range. As shown in Fig. 4a, the GEANT4 simulation results suggest that the energy resolution of one electron incidence is less than $8 \%$, and as shown in Fig. 8a, the results of the TKSC irradiation tests indicate that the energy resolution of one electron incidence is also less than $8 \%$. Figure $8 \mathrm{~b}$ presents the resolution of the GSO scintillator energy range. As shown in Fig. 4b, the GEANT4 simulation results show that the energy resolution of one electron incidence is less than $60 \%$. However, as shown in Fig. $8 \mathrm{~b}$, the results of the KURRI irradiation tests show worse energy resolution than that estimated by the GEANT4 simulation under $10 \mathrm{MeV}$. This is due to background gamma rays generated by the facility. As shown in Fig. 7b, the irradiation
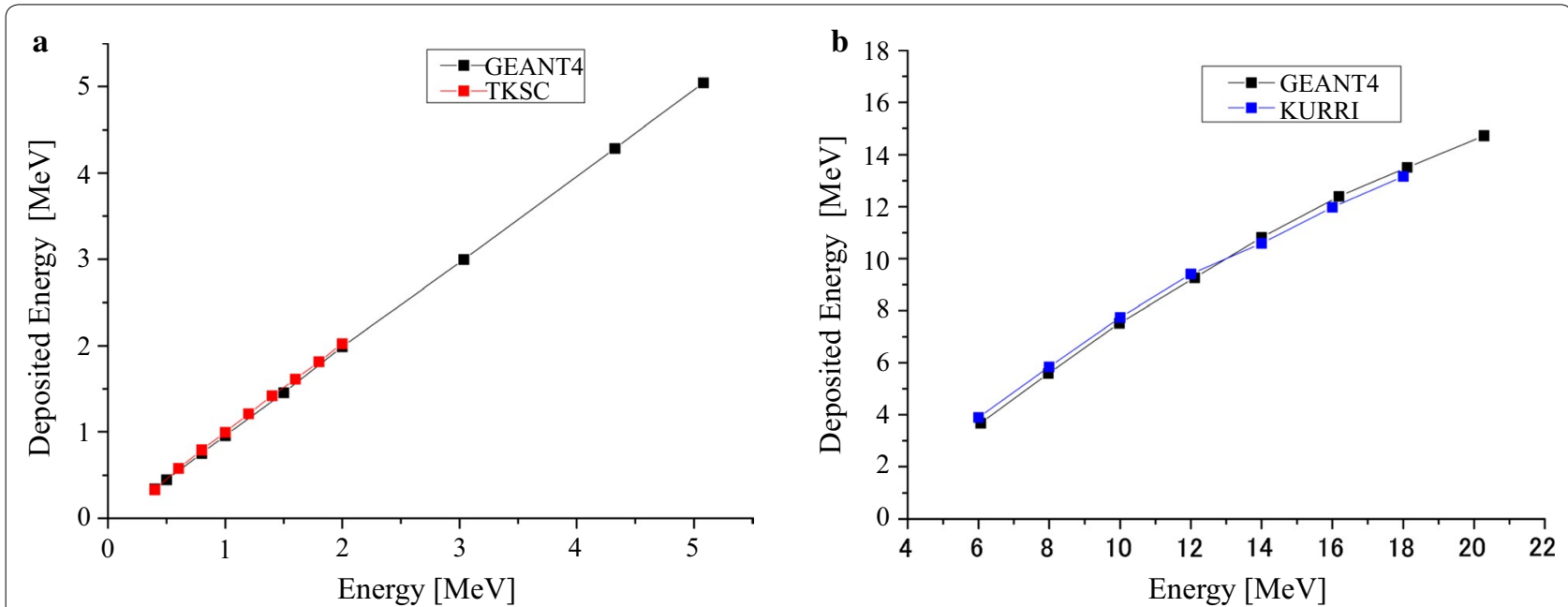

Fig. 7 The deposited energies converted from the ADC channels obtained by the irradiation tests and the deposited energies estimated by the GEANT4 simulation are plotted. a The results of the irradiation tests for $0.4-2 \mathrm{MeV}$ electrons at TKSC (red line) and the GEANT4 simulation results for 0.5-5 MeV electrons (black line). $\mathbf{b}$ The results of the irradiation tests for 6-18 MeV electrons at KURRI (blue line) and the GEANT4 simulation results for 6-20 MeV electrons (black line) 

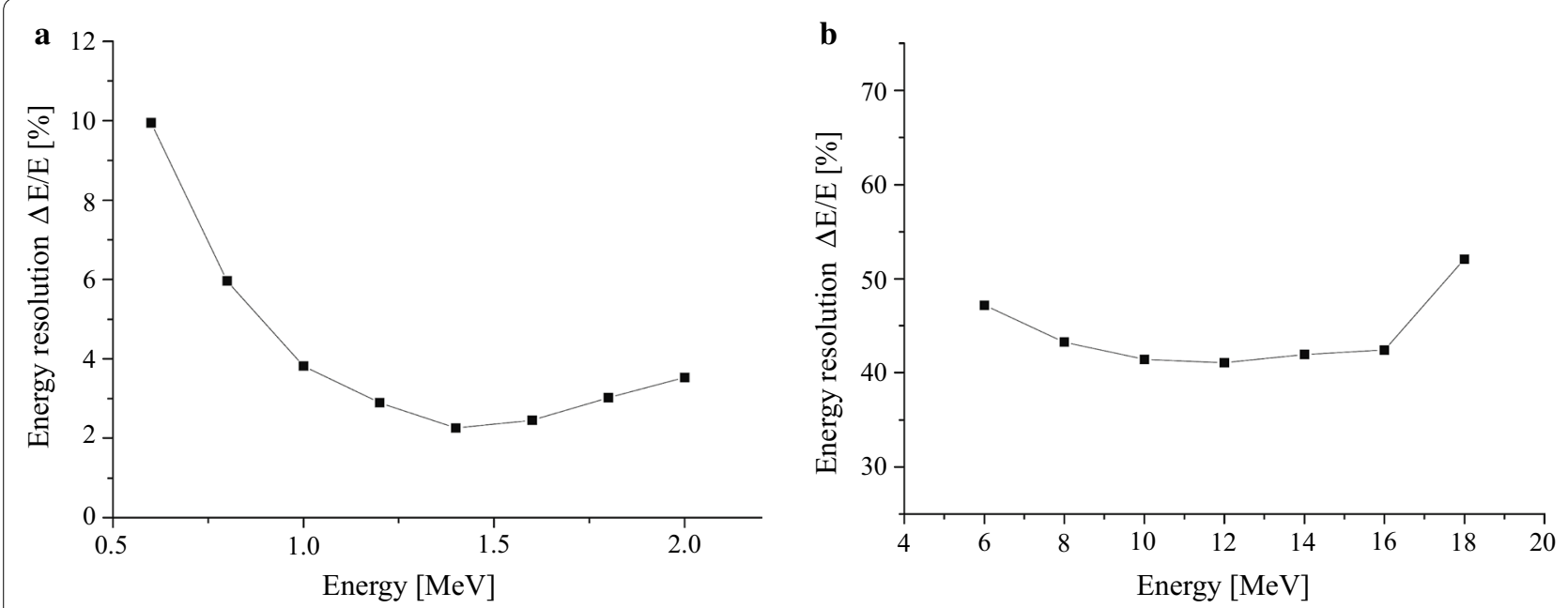

Fig. 8 The energy resolution evaluated by the irradiation tests: a the irradiation test of $0.6-2 \mathrm{MeV}$ electrons at TKSC and $\mathbf{b}$ the irradiation test of 6-18 MeV electrons at KURRI

test result is judged to be appropriate from the tendency that the peak channel results match each other, and the resolution is $60 \%$ or less in considering the influence of the background gamma rays at the facility.

We examined the effects of protons and electrons coming from the side of the XEP. For the experiment of the proton irradiation, the cyclotron facility at the National Institutes for Quantum and Radiological Science and Technology (QST) was used. The irradiation test results confirm that the anti-scintillator detects electrons at $E>11 \mathrm{MeV}$ and protons at $E>41 \mathrm{MeV}$. It is also confirmed that particles influencing the observation are physically stopped by both the 9-mm-thick aluminum shield and the 1-mm-thick tantalum shield. As results of the preflight tests noted above, we concluded that the XEP works well as designed.

\section{Test of calibration signals}

Figure 9 shows a result of an in-flight test of calibration signals. As shown in Fig. 9, the peak positions of each channel between the ground calibration and the in-flight calibration show in good agreement. The in-flight counts of SSD-2 appear at other channels because natural electrons in space are detected. As a result of the in-flight calibration, the voltages applied to the sensors, all electronic circuits and all sensors of the XEP show nominal status
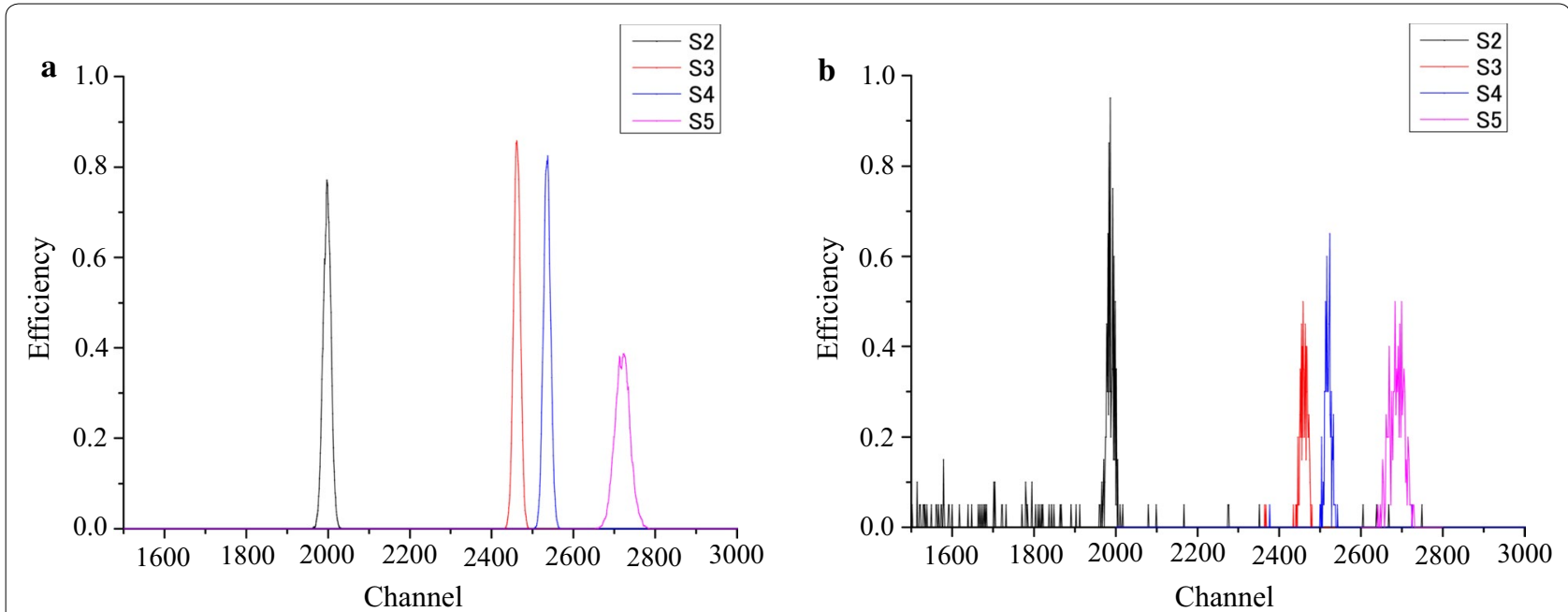

Fig. 9 a The result of the ground experiment inputting the calibration signals at TKSC and $\mathbf{b}$ the result of the in-flight experiment inputting calibration signals on the orbit 
as expected, and thus, we concluded that the XEP instrument is overall in good shape.

\section{Initial observation results}

The XEP has successfully observed variations of relativistic electrons after completing its commissioning phase in March 2017. Here, we show an initial XEP result obtained during a small magnetic storm on April 4, 2017. The top two panels in Fig. 10 show $L$-value versus time $(L-T)$ diagrams for $0.9 \mathrm{MeV}$ and $2.5 \mathrm{MeV}$ electrons. Note that the $L$-shell used in this study follows the definition of the McIlwain $L$-parameter derived from the IGRF model. The small storm is identified in the Dst index, and the peak Dst $=-44 \mathrm{nT}$ was observed at $12 \mathrm{UT}$ on April 4, 2017. Before the small storm began, the flux of the outer belt was high due to large flux enhancement during the long-lasting recovery phase of the magnetic storm on March 27, 2017.

After a signature of the storm commencement at 4 UT on April 4, 2017, the fluxes of relativistic electrons disappear, especially when the Dst index shows a dip signature of the main phase. The disappearance of electrons was observed at $L>3.9$ for $0.9 \mathrm{MeV}$ and $L>3.8$ for $2.5 \mathrm{MeV}$ electrons. During the recovery of the small storm up to April 6, the electron fluxes of both energies in the inner region of the outer radiation belt recovered, while the fluxes of the outer part of the outer radiation belt did not recover from the electron flux decrease during the small storm. Indeed, large flux enhancement in the outer region of the outer radiation belt was not expected due to the low solar wind speed and strongly northward interplanetary magnetic field (IMF) $\mathrm{B}_{\mathrm{z}}$ orientation during this interval (Reeves et al. 2011; Miyoshi et al. 2013).

Figure 11 shows an energy versus time $(E-T)$ diagram of electrons from 0.4 to $5.4 \mathrm{MeV}$ for 2 days of April 2 and 3, 2017. This figure shows that the data at each energy are normally acquired without any problem. The XEP also observed that differential electron fluxes of each energy range tend to decrease as the spacecraft moves away from the Earth. These results provide a good example of the energy, time and spatial dependent variations of high-energy electron fluxes in the outer radiation belt during magnetic disturbances. As shown in this initial observation, the XEP will provide us with high-quality information for better understanding the dynamic variation of the radiation belts in response to solar wind.

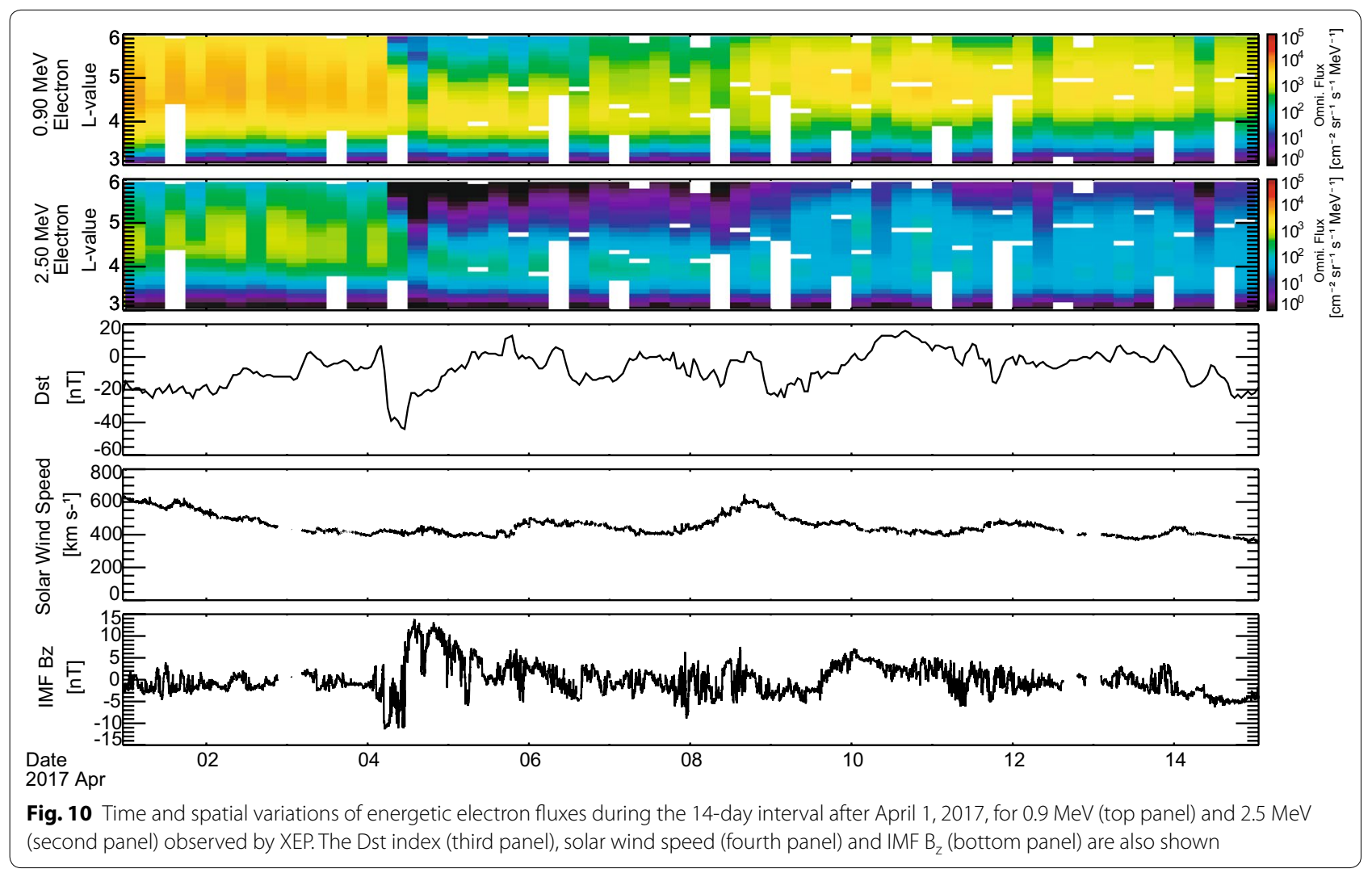




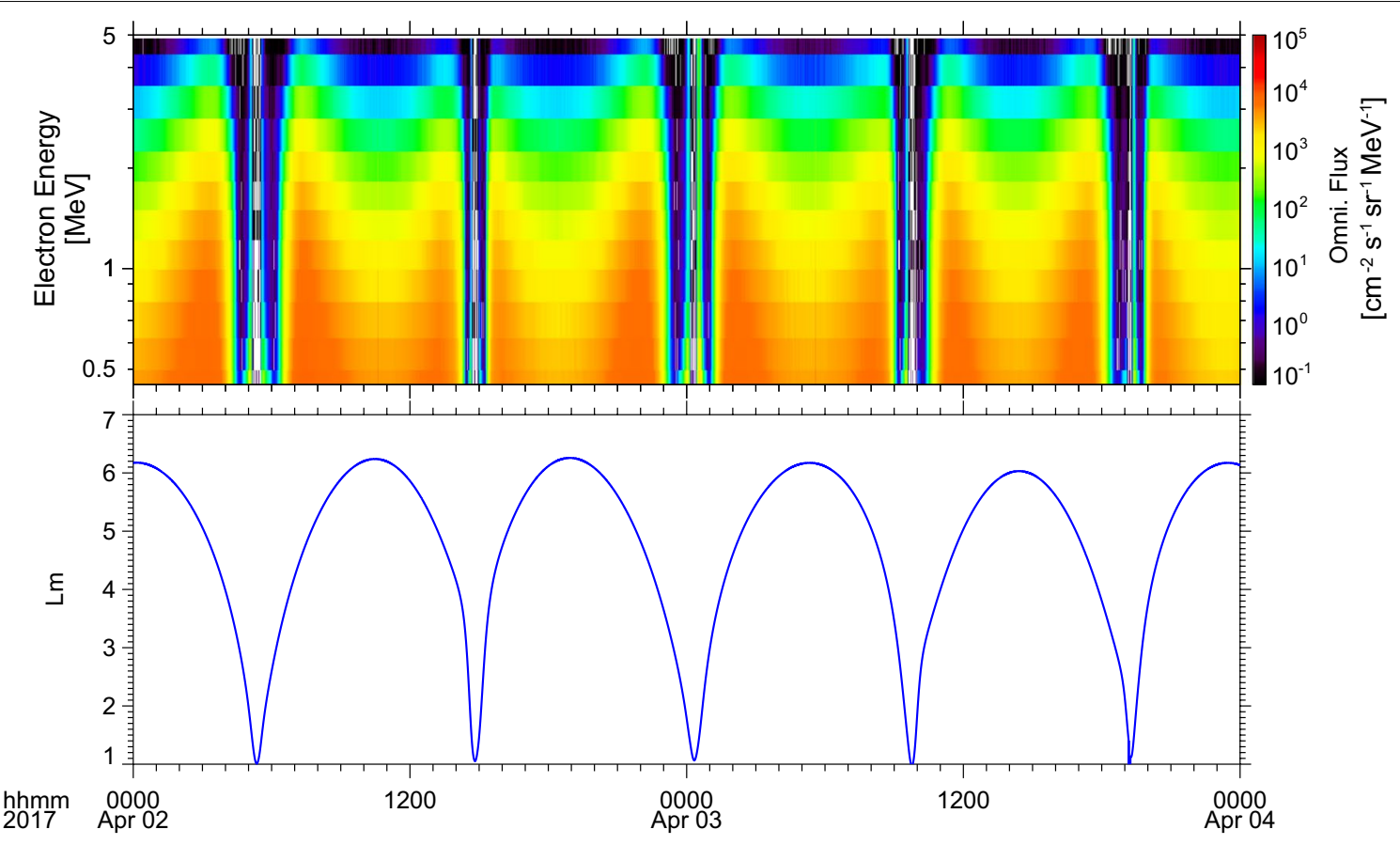

Fig. 11 An energy-time diagram of energetic electron fluxes observed by XEP during the two-day interval after April 2, 2017. The bottom panel shows the Mcllwain $L$-parameter $\left(L_{m}\right)$ for a pitch angle of $90^{\circ}$

\section{Summary}

The XEP is responsible for measuring the highest energy part of the whole energy range covered by four electron sensors onboard Arase, and it can precisely measure relativistic electrons up to $20 \mathrm{MeV}$ to satisfy the mission requirements. The XEP consists of the five SSDs and the GSO scintillator, and it is carefully designed to eliminate the contamination from protons, heavy ions and higherenergy electrons. The XEP measurement is essentially necessary to address physical mechanisms of electron acceleration and loss in the outer radiation belt.

The XEP has initiated high-energy electron measurements in the Earth's radiation belts since March 24, 2017. We confirmed that the XEP achieves the expected observation performance as designed, and the XEP has successfully captured dynamic variations of relativistic electron fluxes in the radiation belts during magnetic storms. The XEP is providing us with information on the behavior of $\mathrm{MeV}$ range electrons. The measurement of the XEP will contribute to better understanding of physical mechanisms behind such dynamic variation of the radiation belts.

Since the energy range of the XEP $(0.4-20 \mathrm{MeV})$ measurement overlaps that of HEP $(0.07-2 \mathrm{MeV})$, cross-calibration between the XEP and the HEP is possible. We expect that the cross-calibration enables us to connect both energy spectra seamlessly.
Such continuous energy spectra in the radiation belts will greatly contribute to the progress of studies on the cross-energy coupling process in the inner magnetosphere.

\section{Authors' contributions}

$\mathrm{NH}$ is the PI of XEP. TT supports to carry out the irradiation test and to calibrate the XEP data. IS supports to analyze initial observation results of the XEP. HM supports to develop the design of the XEP. All authors read and approved the final manuscript.

\section{Author details}

${ }^{1}$ Research and Development Directorate, Japan Aerospace Exploration Agency, Tsukuba 305-8505, Japan. ${ }^{2}$ Graduate School of Science, The University of Tokyo, Tokyo 113-8654, Japan. ${ }^{3}$ ISAS/JAXA, Sagamihara 252-0222, Japan.

\section{Acknowledgements}

The authors wish to thank Meisei Electric Co., Ltd. (Y. Terakado, M. Nakazawa, H. Takara, S. Fujishima, T. Omoto, S. Mizumoto, C. Hatakeyama, K. Tanaka and Y. Koumoto) and FAM Science Co., Ltd. (M. Fujii and A. Fujii) for their technical assistance regarding this instrument. In particular, the authors would like to acknowledge K. Asamura, T. Mitani and T. Mukai from ISAS/JAXA for their valuable technical comments. The authors would also like to thank T. Takahashi and N. Abe at the Kyoto University Research Reactor Institute (KURRI-LINAC) for their support in calibrating this instrument (6-18 MeV electrons), as well as the National Institutes for Quantum and Radiological Science and Technology (70 MeV/n protons) and T. Kaneko, H. Otomo, T. Hirose and T. Hirao from Ryoei Technica Corporation (0.4-2 MeV) for their support in calibration. Finally, the authors would like to thank M. Teramoto, S. Kurita, Y. Miyoshi and T. Hori from the ERG Science Center for their support in data analysis, and K. Seki from the University of Tokyo for her valuable comments. The XEP data processing was partly supported by the SEES/JAXA. The OMNI data for CDAWeb and the Dst for Kyoto University. 


\section{Competing interests}

The authors declare that they have no competing interests.

\section{Availability of data and materials}

XEP data of the ERG project are distributed by the ERG Science Center (https:// ergsc.isee.nagoya-u.ac.jp/index.shtml.en)

\section{Funding}

Not applicable.

\section{Publisher's Note}

Springer Nature remains neutral with regard to jurisdictional claims in published maps and institutional affiliations.

Received: 20 May 2018 Accepted: 1 August 2018

Published online: 16 August 2018

\section{References}

Ebihara Y, Miyoshi Y (2011) Dynamic, inner magnetosphere: a tutorial and recent advances. In: Liu W, Fujimoto M (eds) The dynamic magnetosphere, pp 145-187. https://doi.org/10.1007/978-94-007-0501-2_9

Hikishima M, Kojima H, Katoh Y, Kasahara Y, Kasahara S, Mitani T, Higashio N, Matsuoka A, Miyoshi Y, Asamura K, Takashima T, Yokota S, Kitahara M, Matsuda S (2018) Data processing in the software-type wave-particle interaction analyzer on board the Arase satellite. Earth Planets Space. https://doi.org/10.1186/s40623-018-0856-y

Katoh Y, Kojima H, Hikishima M, Takashima T, Asamura K, Miyoshi Y, Kasahara Y, Kasahara S, Mitani T, Higashio N, Matsuoka A, Ozaki M, Yagitani S, Yokota S, Matsuda S, Kitahara M, Shinohara I (2018) Software-type wave-particle interaction analyzer on board the Arase satellite. Earth Planets Space 70(1):4. https://doi.org/10.1186/s40623-017-0771-7
Mitani T, Takashima T, Kasahara S, Miyake W, Hirahara M (2018) High-energy electron experiments (HEP) aboard the ERG (Arase) satellite. Earth Planets Space 70(1):77. https://doi.org/10.1186/s40623-018-0853-1

Miyoshi Y, Kataoka R (2005) Ring current ions and radiation belt electrons during geomagnetic storms driven by coronal mass ejections and corotating interaction regions. Geophys Res Lett 32:L21105. https://doi. org/10.1029/2005GL024590

Miyoshi Y, Morioka A, Obara T, Misawa H, Nagai T, Kasahara Y (2003) Rebuilding process of the outer radiation belt during the November 3, 1993, magnetic storm-NOAA and EXOS-D observations. J Geophys Res. https ://doi.org/10.1029/2001ja007542

Miyoshi Y, Kataoka R, Kasahara Y, Kumamoto A, Nagai T, Thomen MF (2013) High-speed solar wind with southward interplanetary magnetic field causes relativistic electron flux enhancement of the outer radiation belt via enhanced condition of whistler waves. Geophys Res Lett. https://doi. org/10.1002/grl.50916

Reeves GD, Morley SK, Friedel RHW, Henderson MG, Cayton TE, Cunningham G, Blake JB, Christensen RA, Thomsen D (2011) On the relationship between relativistic electron flux and solar wind velocity: Paulikas and Blake revisited. J Geophys Res. https://doi.org/10.1029/2010JA015735

Shprits YY, Elkington SR, Meredith NP, Subbotin DA (2008a) Review of modeling of losses and sources of relativistic electrons in the outer radiation belt I: radial transport. J Atmos Solar Terr Phys 70:1679-1693

Shprits YY, Subbotin DA, Meredith NP, Elkington SR (2008b) Review of modeling of losses and sources of relativistic electrons in the outer radiation belt II: local acceleration and loss. J Atmos Solar Terr Phys 70:1694-1713

Summers D, Thorne RM, Xiao F (1998) Relativistic theory of wave-particle resonant diffusion with application to electron acceleration in the magnetosphere. J Geophys Res 103:20487-20500

Yasutomo S, Matsumoto H, Obara T (2009) Preliminary result of radiation measurement on GOSAT. Paper 2009-r-2-23 presented at proceedings of the 27th international symposium on space technology and science (ISTS), Tsukuba, Japan, 5-12 July 2009

\section{Submit your manuscript to a SpringerOpen ${ }^{\circ}$ journal and benefit from:}

- Convenient online submission

- Rigorous peer review

- Open access: articles freely available online

- High visibility within the field

- Retaining the copyright to your article

Submit your next manuscript at $>$ springeropen.com 